\title{
Optical Diagnostic in Medicine — Needs and Possibilities
}

\author{
J. Kasprzak*, D. SAmsel, A. Borkowska And M. KeCiK \\ Department and Ophthalmology Clinic, I Medicine Faculty, Medical University of Warsaw \\ W. Lindleya 4, 02-005 Warsaw, Poland
}

\begin{abstract}
Methods of optical imaging used in medical diagnostic (with taking the newest diagnostic techniques into consideration in particular) have been presented. Selected optical characteristics of tissues having an influence on the range of the classical methods of the optical imaging have been discussed in detail. The respond to the classical method limitations was to use scanning methods and detection techniques consisting in three-dimensional imaging of tissue structures in real time. A specific eye structure allows the wide using of these techniques in ophthalmology. Such advantages like non-invasiveness, non-contactness and high resolution (guaranteeing the safety of a patient) show a huge success of these techniques. The results of the research (conducted in many research centers) point at the wider application possibility of optical scanning method in medical diagnostic.
\end{abstract}

PACS: 42.30.Wb, 87.57.-s, 42.79.-e, 42.79.Qx

\section{Introduction}

Optical diagnostic occupies a very important place in medical science. Besides the classical optical methods, the number of techniques applying among other things ultrasounds, $\mathrm{X}$ radiation, magnetic nuclear resonance or positon emission topography are used $[1,2]$.

Specific light features allow one both the structures imaging and the dynamic evaluation of the processes within tissues. In medical applications, the analysis of spatial and time dependences of the optical radiation permits revealing the characteristics which are impossible to achieve by means of other methods. The joining of spectral and polarimetric measurements with the methods of spatial localization allows creating maps of a specific size used for tissue diagnostic. Like in the diagnostic with the use of $\mathrm{X}$ radiation, classical ultrasounds or magnetic resonance a very important thing in structural imaging is the visualization possibility of the tissue anatomy. In these methods the dominant problem is the tissue discrimination with considering their boundary identification within internal structures in particular.

The aim of the functional imaging is the visualization of the property changes of the tissues accompanying metabolism (flow of body fluid, intratissue oxygen consumption) $[3,4]$. Particularly functional properties of optical methods such as non-invasiveness and specific interactions with tissues have always aroused a great interest. Therefore, along with the rapid optoelectronics development, there appeared a lot of new techniques of optical imaging [5-9].

\footnotetext{
* corresponding author; e-mail: kasprzak@amwaw.edu.pl
}

\section{Light propagation in tissues}

The multicomponent tissue structures of different order state (intracellular structures, cells and conglomerates of cells) make up the scattering centers with dimensions including a wide values range. As a result, the light interaction with biological tissues becomes a very complex process.

The average value of the tissue refractive index is higher than the air refractive index which is the cause of the Fresnel light reflection on the boundary: tissueair. Depending on the tissue type, about $2-4.6 \%$ light is reflected from the boundary surface. The rest of the light penetrates the tissue being scattered and absorbed. The optical characteristics of the scattering medium are described by means of the extinction coefficient combining absorption with scattering $[10,11]$.

The Lambert-Beer law describes the situation when the collimated light beam passes through tissues. In mediums characterized by high optical density (as a result of many interactions with scattering centers) the beam totally fades just after traversing a distance of a few millimeters far into a tissue. When the scattering is a dominated process, the significant part of the radiation is backscattered.

The absorption coefficient characterizes the absorption process. The light absorbed can be converted into the heat which is absorbed in photochemical reactions or radiated back at different frequency in a fluorescence process. In biological samples the absorption spectrum depends on the characteristics of dominant components and on water contest in a tissue. The refractive index of the majority of tissues equals about 1.4. Its value may change from about 1.33 (for high hydration tissues, e.g. vitreous body) to 1.55 (for the concentrated protein solution and 
the adipose tissue) [10, 11]. The typical values of the absorption coefficient in biological tissues range widely from $10^{-2}$ to $10^{4} \mathrm{~cm}^{-1}$. In the ultraviolet region and the infrared region above $2 \mu \mathrm{m}$, the penetration depth of continuous wave corresponds to the thickness of the individual cellular layers (Fig. 1A) [12]. In the range of $0.3-0.6 \mu \mathrm{m}$ a typical penetration depth equals from 0.5 to $2.5 \mathrm{~mm}$. Here, the scattering and absorption phenomena occur to the same extent. About 15-40\% of the incident light is included in the reflected and scattered fluxes (Fig. 1B). In the range of $0.6-1.5 \mu \mathrm{m}$ the scattering is much higher than absorption and the penetration depth increases to $8-10 \mathrm{~mm}$. In this range due to high backscattering, the intensity of the reflected and backscattered light may increase even to $70 \%$ of the incident light (Fig. 1C) [10, 11].
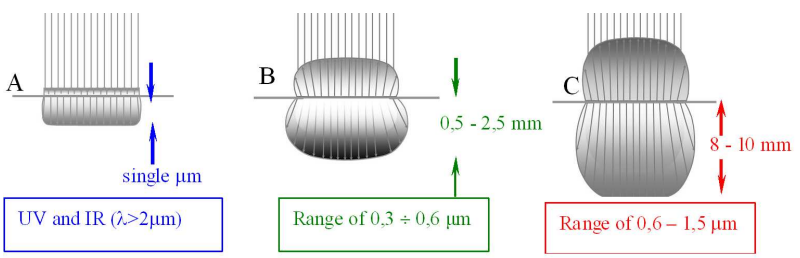

Fig. 1. The influence of the value of scattering and absorption coefficients on light penetration in tissues - a scheme.

As a result of backscattering in multiplayer and multicomponent biological structures the individual components of the light reflected from different layers located on the beam path mix. The individual tissue structures include components of different spectral characteristics (melanin, bilirubina, hemoglobin). The selective absorption of each of these components additionally complicates the analysis of the interaction process of the light with tissues [9].

\section{Selected features of classical imaging systems}

The spectacular invention speeding up the progress of the optical imaging theory was the photography done by Daguerre in 1839. Historically, the optical imaging theory in geometrical approach was created the earliest [13-15]. This theory uses the notion of an optical ray resulting from the approximation assuming that the length of the electromagnetic wave is small and it can be neglected. It is assumed that each point of the object is a source of light rays which after passing through the optical system should be focused in one point.

The analysis results of the optical imaging in wave and geometrical approach are similar as long as the optical system can be treated as unlimited and aberrationless. After detection the image should have a form possible for interpretation, containing various characteristics such as shape characteristic, colour characteristic, surface character characteristic, etc.
The progress in the field of electronic techniques of the measurement and numerical methods speeded up the development of the methods using the point detection. In these techniques a detector scans an image area, however, during the scanning there is the need of the performance of additional analyses. This makes the whole reconstruction time significantly longer than in case of standard methods. The receivers used in standard methods in some cases can be treated as ordered collections of individual detectors [15]. However, there are significant differences both in the range of technical solutions (optical systems, illuminators, detector sensitivity, etc.) and algorithms leading to image reconstruction.

The possibility of the further development of imaging methods using conventional optics is gradually being exhausted. In these systems the three-dimensional images are imaged with the use of two-dimensional detectors. The optical signal contains the information not only from the object plane but also from reflecting structures both in front of and behind this plane. The light scattered in the structures lying on the beam axis overlays the radiation coming from the object plane causing the decrease in the contrast and the resolution of the image examined. To eliminate this shortcoming special detection techniques were searched; sensitivity and resolution were increased. A number of imaging methods permitting the imaging of the axial cross-sections of the object examined were worked out. A good example can be here the computer tomography $(\mathrm{CT})$. In this method on the basis of the one-dimensional measurement series the axial imaging of two-dimensional images (2D) is performed. In the CT method the measurement of the collimated X radiation beam is done after passing through the object examined. The measurement is repeated after the parallel shifting of a source and a detector. After performing a particular number of steps necessary for the whole object scanning, the one-dimensional diagram of the $\mathrm{X}$ -radiation intensity passing through the object examined is achieved. Next, the measuring system is rotated at a known angle and the measuring sequence is repeated $[16,17]$. The 2D image reconstruction consists in the determination of the spatial distribution of the light beam attenuating coefficient - on the basis of the radiation intensity value obtained in the next measuring sequences. The three-dimensional reconstruction is reduced to multiple repetition of the $2 \mathrm{D}$ procedure in the axis direction perpendicular to the scanning plane [18-20].

The optical tomography (OT) in its basic version uses similar rules of the image reconstruction [21-23]. The basic advantage of this method is the possibility of using the optical radiation in the range from the visible to the near infrared. In a wide frequency range the light is relatively safe - even for high values of the radiation intensity. With preserving the safety standards for the sources of optical radiation the optical tomography is a fully non-invasive diagnostic method. The devices of this type can be applied both in regular screening and continuous pathological changes monitoring. 


\section{Optical scanning methods in medical imaging}

The full non-invasiveness of the optical scanning methods is an exceptionally attractive feature for medical applications. Simultaneously, the perspective of breaking many limitations of the classical optical imaging methods makes it possible to use this technique (in connection with classical imaging methods) in many branches of medicine for intravital visualization of internal optical structures occurring in tissues with various optical characteristics. Besides the optical tomography (developing very fast in the last decade) many works applying the specific possibility of optical scanning methods were worked out [24-26]. Particularly, many of these solutions were developed for the need of ophthalmologic diagnosis. Commercial versions of the devices using the optical scanning methods include among other things confocal ophthalmoscopes, polarimeters, retina thickness analyzers, Doppler's flow meters and optical coherent tomographs.

\subsection{Optical scanning methods analysis used in ophthalmology}

The crucial moment of the optical scanning development turned out to be the application of this method by Petren and Hadravsky in 1967. They used these methods for internal live tissue structures examination. The first clinical tests of ophthalmoscopes started at the beginning of the 80s. On the European market the devices of this type appeared in the second half of the 80s [28]. However, much more dynamic development of the devices using the optical scanning methods was only observed in the last decade of the 20th century. During a few years ophthalmologists have received many various offers using confocal technique of the devices making the blood flow examination, fluoroangiography, the retina topography examination or the nerve fiber analysis possible [27-31].

\subsection{Confocal method}

The dynamic development of optical scanning methods used in ophthalmology gave birth to confocal, scanning laser ophthalmoscopes. The measurement principle requires that a source and a single detector have the same focus [32-34]. Well known dependences of reverse proportionality of spatial resolution and visual field are utilized. The visual field is limited by applying, on the one hand, the light source focused on small tissue area and, on the other hand, the objective focusing the light (reflected from the tissue) on a single detector (with the same focus as the lens of the illuminator) (Fig. 2). In this focus configuration, the light scattered in the surface located in front of and behind the focal plane is effectively eliminated. As a result of this procedure, the expense of the visual field limitation, the resolution and image contrast increase. In order to increase the visual field, the transverse scanning method is applied. Here, the measurements are performed by moving the measurement beam or the object examined together with the detector. This technique allows measurement performance in points uniformly distributed within the area examined. Thanks to that, it is possible to achieve the tables of numerical values (corresponding to these points) characterizing optical characteristics used in the process of digital analysis for the two-dimensional image reconstruction of the tissue examined.

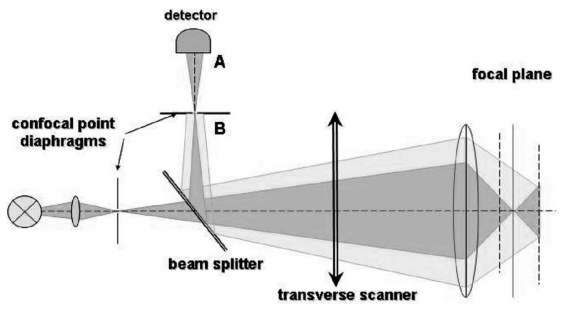

Fig. 2. A diagram of a confocal method. (A) The detection in a situation when the plane reflecting the radiation is located in the same focus of the illuminator and the detector; (B) the radiation intensity (recorded by the detector) reflected from the plane located in front of or behind the focal plane and limited by the aperture.

The optical scanning method used in contemporary ophthalmologic scanning ophthalmoscopes requires performing a large number of measurements. In the first mass-productive devices of this type the image reconstruction with dimensions of $512 \times 512$ points required performing over 260 thousands measuring cycles.

\subsection{Digital angiography}

Initially the confocal laser ophthalmoscopes were treated as digital funduscameras [35]. The rules of confocal detection in connection with the specific characteristics of the laser radiation were used among other things in devices designed for digital angiography. The selective excitation of small areas with the use of monochromatic laser radiation allows recording the contrast angiographs characterized by high resolution [36-38]. The advantages of this technique appear in case of the indocyanine angiography (ICG). In comparison with conventional techniques the images obtained have much better resolution. The possibility of the simultaneous performing of the fluorescent angiography (AF) and ICG angiography is extremely useful. The recording speed strongly increases to 20 angiographic images per second or to 12 pairs of AF or ICG per second whereas in classical methods the recording speed is limited to single images per second. The digital form of the angiographs creates a new possibility as far as angiographic examination results are concerned.

\subsection{Retina tomography}

Using modifications of the specific features of the confocal technique the devices designed for retina topography evaluation were developed [39-41]. To this aim the laminar scanning is performed. The basis of this technique is performing a series of two-dimensional images during the axial shifting of the focal plane. In confocal detection systems the surface illuminated by a scanning 
beam increases along with an increase in the distance from the focus. Since the image of the surface examined is projected on the detector diaphragm characterized by small aperture (in laminar scanning process), the brightness of individual points changes depending on the location with respect to the focal plane and reaches the maximum value when the element examined is in the beam focus (Fig. 3). On the basis of the corresponding points (in the scans) from consecutive layers it is possible to gain information allowing determining the location coordinate of individual elements in the beam axis direction. The maximum intensity value permits describing the optical characteristics of the tissue examined. Such modified measurement method makes it possible to obtain the simultaneous retina image and topographic maps of its surface.

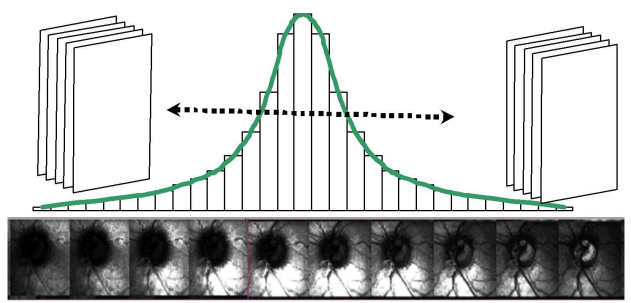

Fig. 3. The intensity change of the light reflected from the retina element recorded by means of the confocal detection system depending on the location with respect to scanning beam focus.

This technique is used for the quantitative analysis of the intraocular nerve hollow as well as holes and retina tears [42-44].

\subsection{Nerve fiber measurement with the use of a polarimetric method}

In laser polarimeters the measurements of the nerve fiber thickness is performed within the area around the optic nerve disc. In this area the nerve fibers radiate creating locally nearly parallel structures. In connection with the specific construction, this layer reveals birefringent characteristics whereas the tissues surrounding are optically inactive. Therefore, this structure has a distinguished optical axis agreeing with the direction of the local fibers system. In practice it means that the light beam polarized according to the nerve fibers direction has a different refractive index in comparison with the beam polarized perpendicularly. After passing through the structure with such characteristics both beams will reveal reciprocal delay proportional to its thickness. This leads to the change of the beam polarization state (Fig. 4).

In the measurements the light polarized in 20 different directions is used. The system counts over the phase shifts recorded at the thickness corresponding to the nerve fiber thickness. Using the polarimetric method it is possible to achieve maps of the fiber thickness within the area around the disk. The fiber nerve thickness dis-

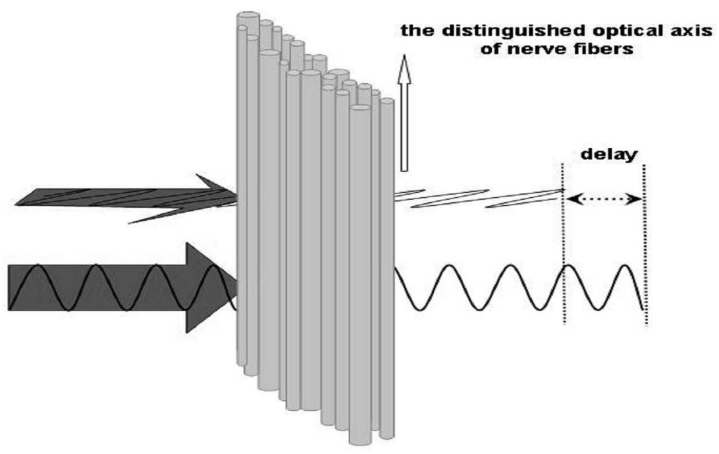

Fig. 4. A principle of operation of the ophthalmologic laser polarimeter. The beam with perpendicular polarization plane is delayed proportionally to the fiber structure thickness.

appearance is a symptom preceding the visual field decrements at patients having glaucoma $[46,47]$.

\subsection{A retina thickness measurement with the use of laser biomicroscopy}

Illuminating transparent tissues of an eye thoroughly by means of a narrow collimated beam, the separated reflections from the consecutive layers are observed. It is frequently used in ophthalmologic effect analysis or in slit lamps. The analysis of the transverse profile of the beam reflected allows evaluating the surface state of individual layers. The distance measurement of the edges of the structures visualized (at a known incidence angle) permits evaluating the axial distance of the reflecting planes [48-50].

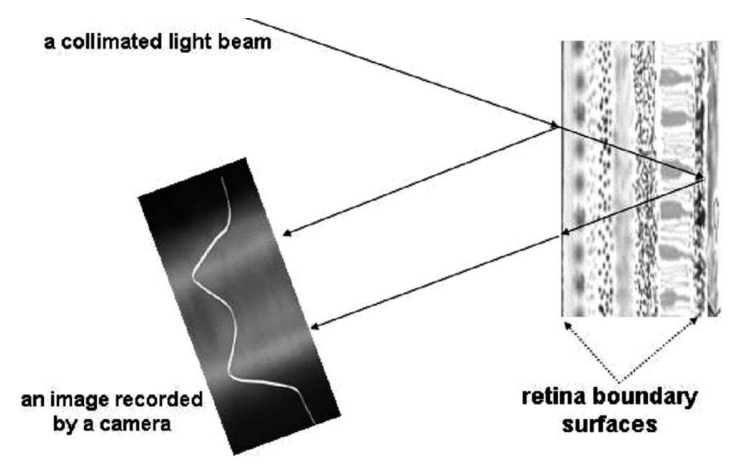

Fig. 5. A principle of the retina thickness measurement with the use of laser biomicroscopy.

This principle is used in optical pachymetry for the measurement of a cornea thickness and the frontal chamber depth. The operation of retina thickness analyzer is based on the slotted method (Fig. 5). In commercially available apparatuses a scanning series including 5-13 regions is performed. In each region 16 sections are recorded when covering the retina image with the dimension of $3 \times 3 \mathrm{~mm}^{2}$. The result is presented in the form 
of a two- or three-dimensional colourful map. The axial resolution equals about $50 \mu \mathrm{m}$. A special algorithm analyzing the refractive index difference in an axial direction also makes the analysis of the nerve fibers thickness possible.

\subsection{Doppler methods}

Scanning laser flowmeter is used for the non-invasive two-dimensional mapping of the perfusion within the area of the eyeball rear pole. For the measurements of the concentration and the erythrocytes moving rate the Doppler effect is used in this device. As a result of the Doppler effect the frequency shift of the wave reflected from the moving object (proportionally to its speed) is observed. It can be written as

$$
\Delta f / f \sim(v \cos \phi) / c,
$$

where $f$ is the wave frequency, $v$ is the object speed, $\phi$ represents the angle between the observation direction and the object velocity $v, c$ - velocity of wave propagation in a medium [51, 52].

In case of caustic waves the ratio $v / c \approx 0.1$ while in case of the light reflected from the erythrocytes the ratio $v / c \approx 10^{-10}$. Such a light frequency change causes the wavelength changes which are not measurable by means of classical optical methods. The measurement of these small shifts makes the use of coherent light possible. Illuminating the erythrocytes and motionless tissues with the use of monochromatic coherent light it is possible to observe two components characterized by insignificantly different frequencies in the beam reflected. As a result of the interference of these components in a detector, the beat effect is observed manifesting itself in the intensity change of the beam (oscillating at the frequency corresponding to the Doppler shift). The oscillations accompanying the beats are a source of a signal characterized by the frequency of a few $\mathrm{kHz}$ order, which does not cause serious problems for accessible detection techniques. The frequency shift resulting from the Doppler effect is the highest during the observation agreeing with the direction of the object movement. During the observation in perpendicular direction $\left(\cos 90^{\circ}=0\right)$ the Doppler frequency shift does not appear. In case of the eyeground observation it causes a problem. The optical system specificity of an eye causes that the light beam reflected directly from the eyeground is almost orthogonal to the retina surface and, as a result, it cannot be used for the measurement of the erythrocytes moving rate. In case of the Doppler shift frequency measurement this limitation can be omitted using the light scattered in the tissue surrounding. Due to multiple reflections in a thin retina layer, part of photons changes its propagation direction and in the next scattering event it reflects from erythrocytes at random angles (Fig. 6). As results from the research, the average value of the Doppler frequency shift is still proportional to the average velocity of erythrocytes moving in a scattering medium and additionally does not depend on the observation direction. Wanting to measure the blood flow velocity in vessels, a certain area of the retina has to be illuminated by a coherent light beam. Next, the intensity of the backscattered light (variable in time) is recorded. After performing the Fourier transform of the signal recorded, the signal image in frequency domain $P(f)$ can be achieved.

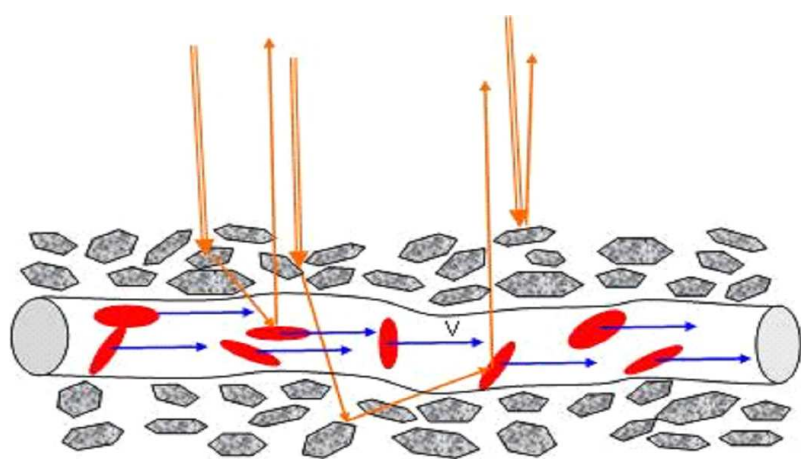

Fig. 6. Detection of the Doppler shift in the eyeground as a result of the backscattered light reflection from erythrocytes in the surrounding tissues.

The signal frequency spectrum shows the distributions of the Doppler shifts formed as a result of the erythrocytes shift within the area illuminated. The local parameters of the flow can be obtained by means of the parameters calculated on the basis of the recorded signal spectrum. The concentration of the moving erythrocytes $(\mathrm{Vol})$ is proportional to the sum of the spectrum values for all frequencies. It can be written as

$$
\text { Vol }=\int P(f) \mathrm{d} f .
$$

Summing the product of power and frequency, the number proportional to the erythrocyte number multiplied by its velocity is obtained. This parameter called the flow (Flw) represents the total shift of all erythrocytes within the measuring area in time unit

$$
F l w=\int P(f) f \mathrm{~d} f .
$$

When dividing the flow by the concentration, the average erythrocytes velocity $(\mathrm{Vel})$ in the area examined is received

$$
\mathrm{Vel}=\mathrm{Flw} / \mathrm{Vol} \text {. }
$$

Using laser scanning technique the measurement series within the rear eyeground pole is performed. Thanks to that, the two-dimensional tables of the flow parameter values can be achieved. After using the coloured encoding, the image of vessels of the area examined is formed.

The Doppler method is the only non-invasive method making the quantitative assessment of blood flow parameters within eyeball possible. In ophthalmology both the Doppler ultrasound and laser flowmeters are used. Laser flowmeters require overcoming many technical problems, however, in comparison with ultrasound devices their spatial resolution is higher. 
The research on using the macular methods for the flow measurement has been conducted recently. The scattering center movement causes the sight shift, speckles which may be used for the blood flow measurement [53-55].

\subsection{Interferometric methods}

One of the devices making use of interferometric methods is the IOL Master used in ophthalmologic biometry. In this device the measurement of the eyeball length is performed by means of the reflectometry method for the radiation of low coherence path $[56,57]$.

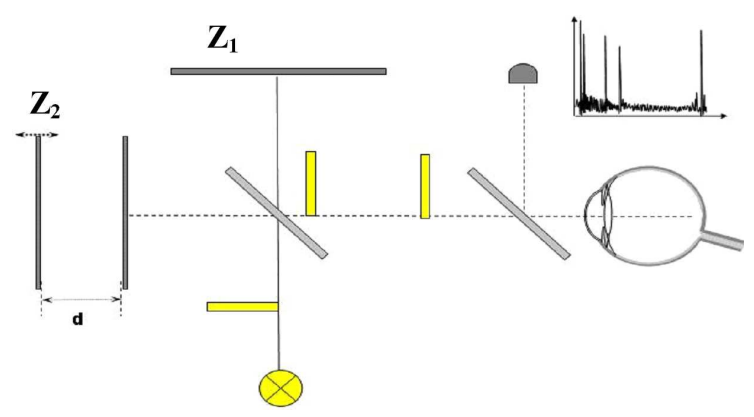

Fig. 7. A diagram illustrating the length measurement using the reflectometry method.

In the device a special interferometric technique using cornea as a reference surface is applied. In the Michelson interferometer the beam of light $(\lambda \approx 855 \mathrm{~nm})$ characterized by high spatial coherence but low time coherence, reflecting from $Z_{1}$ and $Z_{2}$ mirrors, generates the dual system of rays (Fig. 7). Their reciprocal delay results from the optical path difference in the measurement and reference arms. After passing through the interferometer the beam illuminates the eye and then both components are reflected from intraocular surfaces separating the mediums characterized by different refractive indexes. For the determination of the axial length the reflections from the external surface of the cornea and the retina pigmented epithelium are used. When the reference beam delay produced by the movable mirror of the interferometer corresponds to the intraocular optical path, the signal being the result of both beams interference appears. As a result of the recording, the one-dimensional course is obtained. The course resembles the ultrasound signal in A presentation but with much higher resolution. When calculating the geometrical length of the optical path it is necessary to apply the modification taking into consideration the refractive indexes corresponding to intraocular mediums within the measuring beam path. In optical coherent tomography (OCT) like in the IOL Master device the interferometric method is also applied [58-60]. In both techniques the white light interferometers are used. The mirror motion in the reference arm makes the axial scanning of the medium optical characteristics possible. In case of OCT techniques the tomograph image is created as a result of the scanning application realized transversely to the beam axis. The mirror of the transverse scanning (after finishing the measuring cycle in beam axis direction) shifts the beam by steps sampling in the direction perpendicular to the next measuring point. As a result of common presentations of the consecutive axial profiles, the two-dimensional tomogram showing the extinction distribution within the sample examined is achieved (Fig. 8).

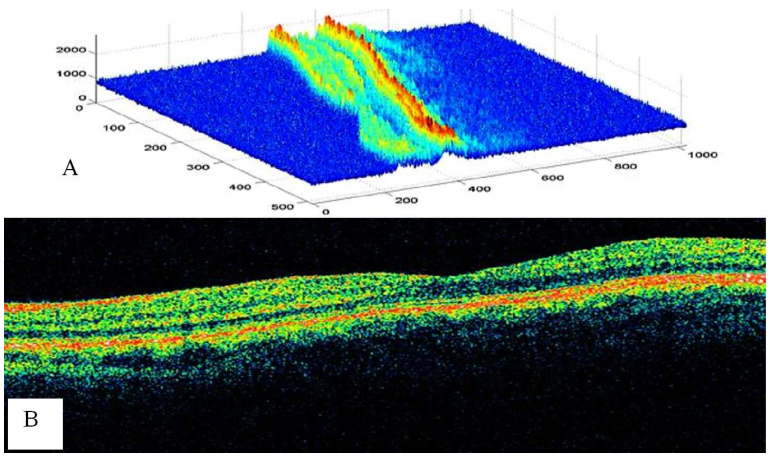

Fig. 8. The scattered radiation distribution obtained for individual points of the scanning line (A). The tomogram showing the image of the internal structure (after the reconstruction with the use of the colour coding) (B).

\section{Perspectives of scanning methods of an optical imaging}

It is necessary to state that optical scanning methods, especially the optical coherent tomography, belong to the group of fast developing diagnostic techniques characterized by high development prospects. Numerous modifications point at bigger and bigger application possibilities in various medical specialties [61-65].

A very important issue is the problem of the propagation (in tissues) of the light emitted in the form of very short pulses. In this case, in the radiation the three components can be distinguished. The intensity value of each component results from the absorption and scattering. For the flat-parallel layers among the passing photons the ballistic photons (coherent components), the photon group characterized by the zigzag trajectory and the component representing the intense scattering can be distinguished [66-69].

The ballistic component (composed of photons whose trajectories run in the direction of the incident radiation) includes photons not being scattered, agreeing with the beam and the photons single-time scattered at small angles. This group of photons is strongly attenuated and occurs in the group of photons passing before the scattering component. The group of photons having the zigzag trajectory includes photons each of which experiences only a few collisions. These photons propagate along the trajectories deviating in low extent from the direction of the incident light and create the earliest fraction 
of the scattering component. The diffusion component is spatially vaster and in more scattering mediums it includes the major photon part participating in many acts of scattering. This group of photons relocates in different directions covering considerably different lengths of the optical paths.

The existing technical possibilities permit the independent detection of the photons arriving the earliest, minimally scattered, which include the geometrical information and the diffusion photons carrying the information about local optical characteristics of the tissue. Currently, the intensive research on the use of the subpicosecond sources of the laser radiation in OCT devices with the resolution allowing imaging of the subcellular structures is conducted [70-72].

The use of the radiation in the range of $1.3 \mu \mathrm{m}$ permits the transclear imaging of the retina structure. Joining the optical scanning techniques gives the possibilities of the simultaneous tomograms performance including the laser ophthalmoscopy or the Doppler analysis of the flows in the retina vessel. However, joining the OCT technique with the ultrasonography technique gives the new possibility of tissue examination excited for oscillation by means of the ultrasound wave. It is a very fast developing branch of the research.

Joining the scanning techniques with different types of catheters and endoscopes finds application in other medical branches, particularly in cardiology. A good example confirming the speed up of the research on the biological structures imaging can be the newest reports concerning issues such as the three-dimensional structure colourful imaging using interferometric methods realized by means of three channels with different radiation wavelength colourful laser ophthalmoscopy allowing imaging both central and peripheral eyeground [73, 74].

Among the newest works put into practice the spectral optical tomography developed by the Team of Medical Physics of Nicolaus Copernicus University in Torun is worth emphasizing. This technique as opposed to the classical OCT method in frequency domain definitely allows speeding up the tomograms recording [75-77]. The solutions applied in this technique bring closer the realization of the long-time expected method of 3D imaging characterized by the resolution of $\mu \mathrm{m}$ and realized in the real time.

\section{References}

[1] C.M. Tempany, B.J. McNeil, J. Am. Med. Assoc. 285, 562 (2001).

[2] C. Doris, Canad. Med. Assoc. J. 153, 1297 (1995).

[3] R. Pittman, B. Duling, Appl. Physiol. 38, 315 (1975).

[4] F. Franhauser, S. Kwasniewska, An. Invest. Baraquer, (Barc.) 24, 293 (1993-94).

[5] N. Scheinfeld, Arch. Dermatol. 140, 473 (2004).

[6] K. Shibuya, H. Hoshino, M. Chiyo, A. Iyoda, S. Yoshida Sekine, T. Iizasa, Y. Saitoh, M. Baba, K. Hiroshima, H. Ohwada, T. Fujisawa, Thorax 58, 989 (2003).
[7] R. Landry, M. Wolffe, C. Burrows, B. Rassow, G. Byrnes, Appl. Opt. 43, 1643 (2004).

[8] A. Mirshahi, J. Buhren, D. Gerhardt, T. Kohnen, J. Cataract \& Refract. Surg. 29, 2295 (2003).

[9] A. Cysewska-Sobusiak, Modelling and Measurement of Biological Signals, Poznań University of Technology, Poznań 2001 (in Polish).

[10] Selected Papers on Tissue Optics: Applications in Medical Diagnostics and Therapy, Ed. V.V. Tuchin, SPIE Press, Bellingham 1994.

[11] F. Schmidt, Ph.D. Thesis, Department of Medical Physics and Bioengineering University College, London 1999.

[12] A. Zajacc, in: Polish Ophthalmology at the Beginning of XXI Century, Ed. T. Kęcik, Oftal, Warszawa 2002, p. 63 (in Polish).

[13] M. Born, E. Wolf, Principles of Optics, Pergamon Press, Oxford 1980.

[14] R. Jóźwicki, Theory of Optical Imaging, PWN, Warszawa 1988 (in Polish).

[15] M. Ostrowski, Optical Imaging, WNT, Warszawa 1992 (in Polish).

[16] E. Rokita, in: Physical Diagnostic Methods in Medical Therapy, Eds. A.Z. Hrynkiewicz, E. Rokita, PWN, Warszawa 2000.

[17] W. Kalender, Phys. Med. Biol. 51, 10 (2005).

[18] Visualization in Biomedical Computing, Eds. K. Hohne, R. Kikinis, Springer-Verlag, Berlin 1996.

[19] S. Arridge, J. Hebden, Phys. Med. Biol. 42, 841 (1997).

[20] N. Gershon, S. Eick, IEEE Comput. Graphics Appl. 17, 29 (1997).

[21] S. Webb, The Physics of Medical Imaging, Adam Hilger, Bristol 1988.

[22] R. Grable, Laser Focus World 32, 113 (1996).

[23] R. Vered, H. Shlomo, H. Taitelbaum, Proc. SPIE 2389, 851 (1995).

[24] I. Ermakov, M. Ermakova, W. Gellermann, P. Bernstein, J. Biomed. Opt. 9, 139 (2004).

[25] L. Rovati, F. Docchio, J. Biomed. Opt. 9, 9 (2004).

[26] M. Klemm, E. Rumberger, A. Walter, G. Richard, Ophthalmology 98, 832 (2001).

[27] G. Zinser, R. Wijnaendts-van-Resandt, C. Ihrig, Proc. SPIE 1028, 127 (1988).

[28] J. Caprioli, J. Miller, Invest. Ophthalmol. Vis. Sci. 29, 1294 (1988).

[29] J. Kasprzak, D. Kecik, T. Kecik, A. Samsel, Okulistyka 1, 7 (1998) (in Polish).

[30] J. Kasprzak, D. Kecik, T. Kecik, A. Samsel, A. Borkowska, Okulistyka 2, 15 (2001) (in Polish).

[31] J. Kasprzak, D. Kecik, T. Kecik, A. Samsel, A. Borkowska, in: Proc. Ist Conf. on Electronics in Medicine, Ed. M. Orłowski, Przemysłowy Instytut Elektroniki, Warszawa 2001, p. 8 (in Polish).

[32] D. Bartsch, M. Intaglietta, J. Bille, W. Dreher, M. Gharib, W. Freeman, Am. J. Ophthalmol. 108, 277 (1989).

[33] G. Zinser, Jpn. J. Vis. Sci. 13, 237 (1992). 
[34] R. Webb, G. Hughes, F. Delori, Appl. Opt. 26, 1492 (1987).

[35] A. Plesch, U. Klingbeil, J. Bille, Appl. Opt. 26, 1480 (1987).

[36] D. Bartsch, R. Weinreb, G. Zinser, W. Freeman, Am. J. Ophthalmol. 120, 642 (1995).

[37] R. Flower, K. Csaky, R. Murphy, Retina 18, 260 (1998).

[38] C. Garcia, M. Rivero, D. Bartsch, D. Ishiko, A. Takamiya, K. Fukui, H. Hirokawa, T. Clark, A. Yoshida, W. Freeman, Ophthalmology 106, 1114 (1999).

[39] A. Azuara-Blanco, A. Harris, K. Cantor, Ophthalmologica 212, 95 (1998).

[40] D. Bartsch, W. Freeman, Neurosci. Biobehav. Rev. 17, 459 (1993).

[41] D. Bartsch, W. Freeman, Exp. Eye Res. 58, 161 (1994).

[42] W. Freeman, D. Bartsch, Australian New Zealand J. Ophthalmol. 21, 139 (1993).

[43] A. Azuara-Blanco, G. Spaeth, J. Nicholl, I. Lanzl, J. Augsburger, Br. J. Ophthalmol. 83, 295 (1999).

[44] A. Anton, N. Yamagishi, L. Zangwill, P. Sample, R. Weinreb, Am. J. Ophthalmol. 125, 436 (1998).

[45] J. Caprioli, R. Ortiz-Colberg, J. Miller, C. Tressler, Am. J. Ophthalmol. 108, 404 (1989).

[46] A. Dreher, K. Reiter, Proc. SPIE 1746, 34 (1992).

[47] J. Caprioli, H. Park, S. Ugurlu, D. Hoffman, Invest. Ophthalmol. Vis. Sci. 39, 2321 (1998).

[48] R. Zeimer, M. Mori, B. Khoobehi, Ophthalmology Vis. Sci. 30, 2099 (1989).

[49] J. Kiryu, Y. Ogura, M. Shahidi, M. Mori, N. Blair, R. Zeimer, Ophthalmology 100, 1040 (1993).

[50] S. Asrani, R. Zeimer, N.S. Goldberger, Ophthalmology 104, 1145 (1997).

[51] G. Feke, D. Goger, H. Tagawa, F. Delori, IEEE Trans. Biomed Eng. 34, 673 (1987).

[52] C. Avila, D. Bartsch, D. Bitner, L. Cheng, A. Mueller, M. Karavellas, W. Freeman, Am. J. Ophthalmol. 126 , 683 (1998).

[53] Y. Aizu, T. Asakura, Proc. SPIE 1431, 239 (1991).

[54] Y. Aizu, T. Asakura, K. Ogino, T. Sugita, Y. Suzuki, K. Masuda, Proc. SPIE 2678, 360 (1996).

[55] J. Valera, A. Doval, J. Jones, Meas. Sci. Technol. 4, 578 (1993).

[56] A. Fercher, K. Mengedhot, W. Werner, Opt. Lett 13 , 186 (1988).

[57] Cr. Hitzenberger, Invest. Ophthalmol. Vis. Sci. 32, 616 (1991).
[58] D. Huang, E. Swanson, C. Lin, J. Schuman, W. Stinson, W. Chang, M. Hee, T. Flotte, C. Puliafito, J. Fujimoto, 'Science 254, 1178 (1991).

[59] A. Ferher, J. Biomed. Opt. 1, 157 (1996).

[60] C. Hitzenberger, W. Drexler, A. Fercher, Invest. Ophthalmol. Vis. Sci. 33, 98 (1992).

[61] G. Tearney, M. Brezinski, B. Bauma, S. Boppart, C. Pitris, J. Southern, J. Fujimoto, Science 276, 2037 (1997).

[62] J. Izatt, M. Kulkarni, H. Wang, K. Kobayashi, M. Sivak, IEEE J. Quant. Electron. 2, 1017 (1996).

[63] X. Li, J. Herrmann, R. Chanta, C. Pitris, W. Drexler, C. Jesser, D. Stamper, D. Golden, M. Scott, J. Fujimoto, M. Brezinski, Proc. SPIE 3598, 152 (1999).

[64] J. Fujimoto, C. Pitris, M. Brezinski, Neoplasia 2, 925 (2000).

[65] C. Pitris, A. Goodman, S. Boppart, J. Libus, J. Fujimoto, M. Brezinski, Instruments Methods 93, 135 (1999).

[66] I. Kwee, Ph. D. Thesis, Department of Medical Physics and Bioengineering University College, London 1999.

[67] C. Klinteberg, R. Berg, S. Linguist, S. Anderson-Engels, S. Svanberg, Proc. SPIE 2626, 149 (2001).

[68] S. Anderson-Engels, R. Berg, A. Persson, S. Svanberg, Opt. Lett. 18, 1697 (1993).

[69] W. Drexler, U. Morgner, F. Kärtner, C. Pitris, Y. Chen, S. Boppart, X. Li, E. Ippen, J. Fujimoto, Opt. Lett. 24, 1224 (1999).

[70] W. Drexler, U. Morgner, R. Ghanta, F. Kärtner, J. Schuman, J. Fujimoto, Nature Med. 7, 502 (2001).

[71] W. Drexler, H. Sattmann, B. Hermann, T. Ko, M. Stur, A. Unterhuber, C. Scholda, O. Findl, M. Wirtitsch, J. Fujimoto, A. Fercher, Arch. Ophthalmol. 121, 695 (2003).

[72] Y. Lingfeng, M. Kim, Opt. Expr. 12, 6632 (2004).

[73] M. Fitzpatrick, J. Am. Med. Assoc. 286, 1567 (2001).

[74] A. Wojtkowski, A. Kowalczyk, P. Targowski, I. Gorczyńska, Opt. Appl. 32, 569 (2003).

[75] M. Wojtkowski, V. Srinivasan, T. Ko, J. Fujimoto, A. Kowalczyk, J. Duker, Opt. Express 12, 2404 (2004); http://www.opticsinfobase.org/ abstract. cfm?URI=oe-12-11-2404.

[76] I. Gorczyńska, A. Szkulmowska, M. Szkulmowski, P. Targowski, J. Kałuzny, M. Wojtkowski, J. Fujimoto, A. Kowalczyk, Proc. SPIE 5861, 1 (2005). 